\title{
Effects of sevoflurane on neuronal cell damage after severe cerebral ischemia in rats
}

\author{
Hee-Pyoung Park ${ }^{1}$, Eun-Ju Jeong ${ }^{1}$, Mi-Hyun Kim ${ }^{1}$, Jung-Won Hwang ${ }^{2}$, Young-Jin Lim ${ }^{1}$, Seong-Won \\ $\mathrm{Min}^{3}$, Chong-Soo Kim ${ }^{3}$, and Young-Tae Jeon ${ }^{2}$ \\ Department of Anesthesiology and Pain Medicine, ${ }^{1}$ Seoul National University Hospital, Seoul, ${ }^{2}$ Seoul National University Bundang \\ Hospital, Seongnam, ${ }^{3}$ Seoul City Boramae Hospital, Seoul, Korea
}

Background: The aim of this study was to investigate the neuroprotective effects of sevoflurane after severe forebrain ischemic injury. We also examined the relationship between the duration of ischemia and neuronal cell death.

Methods: Male Sprague-Dawley rats $(300-380 \mathrm{~g}$ ) were subjected to 6 (each $\mathrm{n}=6$ ) or 10 min (each $\mathrm{n}=10$ ) of near-complete forebrain ischemia while anesthetized with either $50 \mathrm{mg} / \mathrm{kg}$ of zoletil given intraperitoneally or inhaled sevoflurane (2.3\%). Ischemia was induced by bilateral common carotid artery occlusion plus hemorrhagic hypotension (26-30 mmHg). Histologic outcomes were measured 7 days after ischemia in CA1 pyramidal cells of the rat hippocampus.

Results: The mean percentage of necrotic cells in the hippocampal CAl area decreased in the sevoflurane group compared to the zoletil group ( $25 \%$ vs. $40 \%$ after 6 min ischemia, respectively: $\mathrm{P}=0.004$ and $44 \%$ vs. $54 \%$ after 10 min of ischemia, respectively $\mathrm{P}=0.03$ ). The percentage of apoptotic cells was similar in all groups. The percentage of necrotic cells in each anesthetic groups was significantly higher in the $10 \mathrm{~min}$ ischemia group compared to the $6 \mathrm{~min}$ ischemia group ( $\mathrm{P}=0.004$ in the sevoflurane group, $\mathrm{P}=0.03$ in the zoletil group).

Conclusions: The present data show that sevoflurane has neuroprotective effects in rats subjected to near-complete cerebral ischemia. Longer duration of ischemia is associated with more neuronal injury when compared to ischemia of shorter duration. (Korean J Anesthesiol 2011; 61: 327-331)

Key Words: Brain ischemia, Hippocampus, Inhalation anesthetics, Neuron.

\footnotetext{
Received: January 25, 2011. Revised: May 17, 2011. Accepted: May 21, 2011.

Corresponding author: Young-Tae Jeon, M.D. Ph.D., Department of Anesthesiology and Pain Medicine, Seoul National University Bundang Hospital, 300, Gumi-dong, Bundang-gu, Seongnam 463-802, Korea. Tel: 82-31-787-7493, Fax: 82-31-787-4063, E-mail: ytjeon@snubh.org (c) This is an open-access article distributed under the terms of the Creative Commons Attribution Non-Commercial License (http:// creativecommons.org/licenses/by-nc/3.0/), which permits unrestricted non-commercial use, distribution, and reproduction in any medium, provided the original work is properly cited.
} 


\section{Introduction}

Numerous studies have demonstrated some degree of a neuroprotective effect with isoflurane [1,2], sevoflurane $[3,4]$ and desflurane $[2,5]$ after cerebral ischemia. The neuroprotective effects of anesthetic agents depend on the severity of ischemic injury. During less severe ischemia, anesthetic agents (fentanyl, ketamine, and isoflurane) resulted in no differences in outcome. In contrast, in animals sustaining a severe insult, those that were anesthetized with isoflurane had less damage than did rats given either ketamine or fentanyl [6].

Zoletil is a combination of a dissociative anesthetic, tiletamine hydrochloride, and a benzodiazepine, zolazepam hypochloride. Tiletamine is a noncompetitive antagonist at the phencyclidine site of N-methyl-D-aspartate receptor.

Sevoflurane has been shown to provide neuroprotection against focal [3] or incomplete global cerebral ischemia [4] in rats. To our knowledge, there has been no effort to define the effects of sevoflurane against severe forebrain global ischemia. The purpose of the present study was to determine the neuroprotective effects of sevoflurane on severe forebrain ischemic injury. We also examined the relationship between ischemic duration and neuronal death. Neuroprotection was assessed by histopathological evaluation 7 days after ischemia. Degrees of neuronal damage in ischemic hippocampal CA1 cells were assessed by counting necrotic cells after H\&E staining and detection of DNA fragmentation was performed by terminal deoxynucleotidyl transferase-mediated uridine 5'-triphosphatebiotin nick end labeling (TUNEL) staining.

\section{Materials and Methods}

The following study was approved by the Institutional Animal Care and Use Committee. Male Sprague-Dawley rats $(300-380 \mathrm{~g}, 10-16 \mathrm{wks})$ were fasted $12-16 \mathrm{~h}$ before the experiments but were allowed free access to water. Rats were then randomly assigned to one of 4 conditions based on the anesthetics and duration of ischemia. In the $6(n=6)$ or $10 \mathrm{~min}$ $(\mathrm{n}=10)$ sevoflurane group, the animals were anesthetized with $5 \%$ sevoflurane in oxygen and during surgery, a level of $2.3 \%$ sevoflurane in $100 \% \mathrm{O}_{2}$ was maintained under spontaneous breathing. In the $6(\mathrm{n}=6)$ or $10 \mathrm{~min}(\mathrm{n}=10)$ zoletil group, $50 \mathrm{mg} /$ $\mathrm{kg}$ of zoletil (Virbac, Nice, France) was given intraperitoneally. The tail artery was catheterized with a PE-50 catheter to allow continuous recording of arterial blood pressure and blood sampling. The common carotid arteries were encircled with suture. The right jugular vein was cannulated with a silicone catheter for drug infusion and blood withdrawal. A 22-gauge needle thermistor was percutaneously placed adjacent to the skull beneath the temporalis muscle, and pericranial temperature was servoregulated (model TCAT-2 Temperature Controller; Harvard apparatus, Holliston, MA, USA) at $37.5 \pm$ $0.1^{\circ} \mathrm{C}$ by surface heating or cooling. Heparin (50 U) was given intravenously.

Anesthetic conditions were established $30 \mathrm{~min}$ before ischemia, during which time rats in both groups were allowed to stabilize physiologically. Arterial blood gases and hemoglobin were measured $10 \mathrm{~min}$ before and after ischemia.

Transient global ischemia was induced by bilateral common carotid artery occlusion and bleeding to lower the mean arterial pressure (MABP) to $26-30 \mathrm{mmHg}$ using the method originally described by Smith et al. [7]. The carotids were then occluded with aneurysm clips. After 6 or $10 \mathrm{~min}$ of ischemia, the shed blood was reinfused. After regaining consciousness, the animals were maintained in an air conditioned room at $20^{\circ} \mathrm{C}$. For each recovery interval, a set of sham rats was generated $(n=4)$. These animals were exposed to all aspects of sevoflurane anesthesia and surgical preparation for ischemia. The carotids were not occluded, and systemic hypotension was not used.

After completion of the ischemic protocol, rats were allowed to recover for 7 days. All rats were anesthetized with sevoflurane, and the brains were fixed in situ by intraaortic infusion with buffered $10 \%$ formalin. Paraffin-embedded brain sections were serially cut ( $5 \mu \mathrm{m}$ thick) and stained with hematoxylin and eosin. With the investigator blinded to group assignment, injury to the hippocampal CAl sector was evaluated by microscopy. The total number of neurons (viable plus nonviable) was counted for each animal. The percentage of necrotic (eosinophilic) CAl neurons was calculated as necrotic neurons/total neurons $\times 100$. In each animal, five optical fields of the hippocampal CAl region were examined.

For the detection of DNA fragmentation, TUNEL staining was performed with the Apoptag ${ }^{\circledR}$ Peroxidase In Situ Apoptosis Detection Kit S7100 (Millipore Corporation, Billerica, USA). After deparaffinizing with xylene and graded concentrations of alcohol, brain sections were exposed to Proteinase K for 15 min at room temperature. Endogenous peroxidase activity was quenched with $3 \%$ hydrogen peroxide in phosphate buffered saline (PBS) for $5 \mathrm{~min}$ at room temperature. Sections were then incubated with terminal deoxynucleotidyl transferase (TdT) in a humidified chamber at $37^{\circ} \mathrm{C}$ for $1 \mathrm{~h}$. After incubation with anti-digoxigenin-conjugate for $30 \mathrm{~min}$ at room temperature, peroxidase substrate $(0.05 \%$ diaminobenzidine, DAB) was applied to develop color. The specimens were then washed with distilled water and were counterstained with $0.5 \%$ methyl green for $10 \mathrm{~min}$ at room temperature. TUNEL-positive neurons that contained apoptotic bodies were identified as being neurons undergoing apoptosis. The number of apoptotic cells was counted under high-power microscopic magnification $(\times 400)$. In each animal, five optical fields of the hippocampal CA1 
Table 1. Physiological Parameters $10 \mathrm{~min}$ before and after Ischemia

\begin{tabular}{|c|c|c|c|c|c|c|c|}
\hline & & $\mathrm{pH}$ & $\begin{array}{l}\mathrm{PaCO}_{2}, \\
\mathrm{mmHg}\end{array}$ & $\begin{array}{l}\mathrm{PaO}_{2}, \\
\mathrm{mmHg}\end{array}$ & $\begin{array}{l}\text { Hemoglobin, } \\
\text { g/dl }\end{array}$ & $\begin{array}{l}\text { MAP, } \\
\text { mmHg }\end{array}$ & $\begin{array}{l}\text { Blood glucose } \\
(\mathrm{mg} / \mathrm{dl})\end{array}$ \\
\hline \multirow[t]{2}{*}{ Zoletil 6} & Before & $7.33 \pm .04$ & $45 \pm 6$ & $230 \pm 38$ & $12.9 \pm 1.9$ & $96 \pm 30$ & $91 \pm 9$ \\
\hline & After & $7.33 \pm .02$ & $40 \pm 9$ & $195 \pm 39$ & $12.5 \pm 1.4$ & $88 \pm 9$ & \\
\hline \multirow[t]{2}{*}{ Sevoflurane 6} & Before & $7.32 \pm .05$ & $48 \pm 10$ & $250 \pm 72$ & $12.8 \pm 1.4$ & $92 \pm 13$ & $94 \pm 10$ \\
\hline & After & $7.33 \pm .03$ & $40 \pm 11$ & $179 \pm 21$ & $11.4 \pm 1.4$ & $87 \pm 8$ & \\
\hline \multirow[t]{2}{*}{ Zoletil 10} & Before & $7.36 \pm .03$ & $43 \pm 3$ & $240 \pm 39$ & $13.8 \pm 1.6$ & $105 \pm 13$ & $96 \pm 10$ \\
\hline & After & $7.33 \pm .02$ & $39 \pm 3$ & $220 \pm 20$ & $14.0 \pm 1.2$ & $116 \pm 14$ & \\
\hline \multirow[t]{2}{*}{ Sevoflurane 10} & Before & $7.31 \pm .03$ & $48 \pm 4$ & $203 \pm 46$ & $14.0 \pm 1.2$ & $84 \pm 12$ & $92 \pm 10$ \\
\hline & After & $7.34 \pm .04$ & $48 \pm 6$ & $198 \pm 37$ & $13.8 \pm 0.6$ & $92 \pm 11$ & \\
\hline
\end{tabular}

Data are expressed as mean \pm SD.

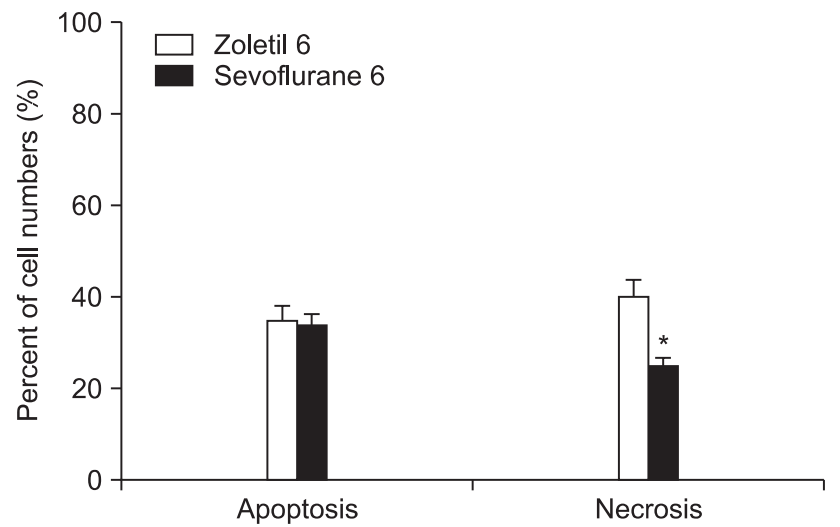

Fig. 1. The percentage of necrotic and apoptotic cells in the hippocampus 7 days after forebrain ischemia. The percentage of necrotic cells was lower in the sevoflurane $6 \mathrm{~min}$ ischemia group $(* \mathrm{P}<0.05)$. There is no significant difference between groups with respect to apoptotic cell number. All data are expressed as mean \pm SD.

region were examined. The percentage of TUNEL-positive cells was defined as the percentage of the number of TUNEL-positive cells to the total cell number.

The quantitative data were presented as the mean \pm SD. Physiologic parameters were analyzed by repeated measures analysis of variance. Statistical significance between groups was analyzed using a Mann-Whitney U test. Differences were considered significant when $\mathrm{P}<0.05$.

\section{Results}

Physiologic parameters are summarized in Table 1. There were no significant differences in mean arterial pressure, $\mathrm{pH}$, $\mathrm{PaCO}_{2}, \mathrm{PaO}_{2}$, and hemoglobin concentration between the control and the sevoflurane treated groups at any time point.

Of the 20 animals receiving $10 \mathrm{~min}$ of ischemia, five rats in the zoletil treated group died before the determination of neurologic damage. A majority of the postoperative deaths occurred within 3 days of ischemia. No animals died after 6 min of ischemia and no neuronal death was observed in the

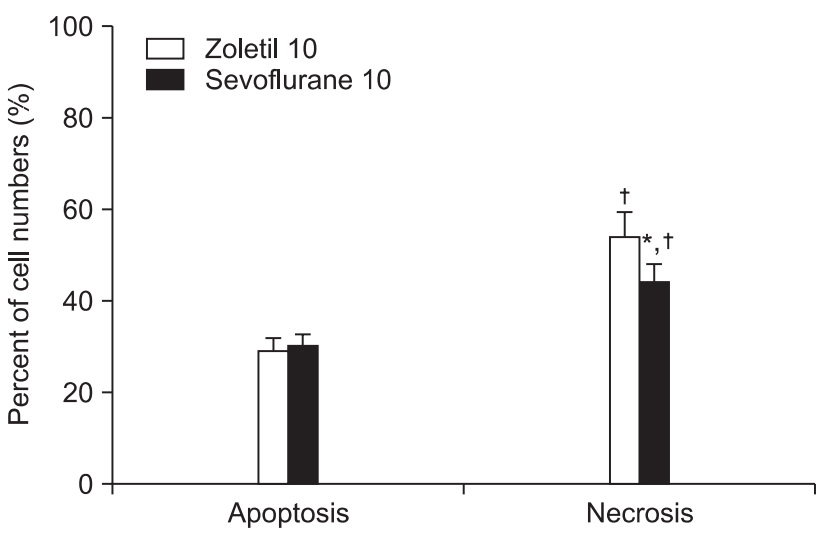

Fig. 2. The percentage of necrotic and apoptotic cells in the hippocampus 7 days after forebrain ischemia. The percentage of necrotic cells was lower in the sevoflurane $10 \mathrm{~min}$ ischemia group $(* \mathrm{P}<0.05)$. The percentage of necrotic cells in each anesthetic group is higher in the $10 \mathrm{~min}$ ischemia group compared with the $6 \mathrm{~min}$ ischemia group $\left({ }^{\dagger} \mathrm{P}<0.05\right)$. There are no significant differences between groups with respect to apoptotic cell numbers. All data are expressed as mean \pm SD.

sham-operated group. The mean percentage of necrotic cells in the zoletil 6 min group was significantly higher than in the sevoflurane 6 min group (Fig. 1: $\mathrm{P}=0.004$ ) and similar results were seen in the zoletil 10 and sevoflurane 10 group (Fig. 2: $\mathrm{P}=0.03$ ). The percentage of necrotic cells in each anesthetic group was significantly higher in the $10 \mathrm{~min}$ ischemia group compared to the $6 \mathrm{~min}$ ischemia group (Fig. 1, 2: $\mathrm{P}=0.004$ in the sevoflurane group, $\mathrm{P}=0.03$ in the zoletil group).

Increases in the percentages of TUNEL-positive cells were observed in all groups as compared to the sham-operated group $(\mathrm{P}<0.001)$. No significant differences in TUNEL-positive cells in the CAl region were observed among the anesthetic groups (Fig. 1 and 2).

\section{Discussion}

In the present study, we investigated the neuroprotective effect of sevoflurane on near-complete cerebral ischemia. This study demonstrated that histologic outcome after 6 or $10 \mathrm{~min}$ 
of severe forebrain ischemia were substantially better in rats with sevoflurane versus zoletil anesthesia 7 days after ischemia. Sevoflurane did not decrease ischemia-induced apoptotic cell death 7 days after ischemia. Shorter duration of ischemia was associated with less neuronal damage.

In rats subjected to incomplete cerebral ischemia, sevoflurane improved neurologic outcomes [4]. In the present study, we focused on the severity of cerebral ischemia. Others have shown that there was no effect of anesthetic agents on outcome in less severe incomplete ischemia [6]. Cerebral blood flow studies after the near-complete insult causing EEG isoelectricity have consistently found flow to be severely reduced. In contrast, examination of cerebral blood flow during less severe forebrain injury found substantial scatter in the magnitude of cerebral blood flow reduction [8]. The near-complete ischemia insult was uniformly severe across all animals. In contrast, findings in the incomplete model varied with respect to concordance between cortical and hippocampal regions as well as between the presence or absence of EEG activity [6]. In the current study, sevoflurane showed better histologic outcomes in rats subjected to severe cerebral ischemia. Six min of ischemia also induced severe neuronal injury. In contrast to our study, a previous study showed sevoflurane completely prevented the neuronal eosinophilic damage (a valid indicator of necrosis) of the brain in incomplete cerebral ischemia [9]. The neuroprotective effect of anesthetic agents seems to be correlated with the severity of neurologic injury. Anesthetic agents provide sustained neuroprotection in the presence of less severe ischemic states $[9,10]$. In contrast, in a rat focal ischemia model, isoflurane did not lead to sustained neuroprotection after 14 days [11]. This suggests that the severity of cerebral ischemia is beyond the neuroprotective potential of anesthetic agents to prevent necrotic cell death.

The mechanisms by which volatile anesthetic agents potentially protect the brain are still unclear. Cerebral protection with sevoflurane has been explained by a reduction in cerebral requirements, similar to barbiturates or isoflurane. The neuroprotective effects of volatile anesthetic agents are associated with decreased apoptotic cell death in the postischemic period [12-15]. Anesthesia with sevoflurane induces a sustained inhibition of neuronal injury by promoting antiapoptotic pathways $[9,16]$. It was demonstrated that sevoflurane prevents the ischemia-induced increase of the pro-apoptotic protein Bax $4 \mathrm{~h}$ after injury induced by incomplete cerebral ischemia [16]. Sevoflurane increases the hippocampal concentration of the anti-apoptotic proteins Bcl-2 and Mdm2 in addition to inhibiting the upregulation of Bax [9]. In the present study, sevoflurane did not decrease TUNEL positive cells. Kawaguchi et al.[13] showed that volatile anesthetics delayed but did not prevent neuronal apoptosis after focal cerebral ischemia. We propose that any anti-apoptotic effect of sevoflurane might disappear 7 days after ischemia.

In the present study, shorter durations of ischemia had significantly less damage than longer durations (i.e. 6 vs $10 \mathrm{~min}$ ). Consistent with our study, the magnitude of ischemic injury is dependent on the duration of ischemia [17]. Sevoflurane did not prevent neuronal damage following ischemia of $6 \mathrm{~min}$ duration. We speculate that even short durations of severe hypotension can cause severe damage which cannot be prevented by sevoflurane.

We used 2.3 vol\% sevoflurane for maintenance of anesthesia. One minimal alveolar concentration of sevoflurane is $2.3 \mathrm{vol} \%$ in adult rats [18]. Therefore, the sevoflurane concentration used in this study was relevant.

There are several limitations in this study. First, the study was limited to 7 days after ischemia. Longer periods of time may be necessary to assess the permanent neuroprotective effect of anesthetics. However, maintaining rats for such a long period of time after cerebral ischemia is not feasible in our laboratory. Second, we did not confirm EEG isoelectricity. However, it is well demonstrated that bilateral common carotid artery occlusion plus hemorrhage (30 mmHg) induces near-complete ischemia in the CA1 region of rats $[6,19]$. Third, we classified necrosis and apoptosis. Attempts to classify CA1 neuronal death as either necrosis or apoptosis may be misleading when attempting to understand mechanisms of death of differentiated neurons. These neurons might possess some of the phenotypic characteristics of necrotic neurons but, in contrast, possess all of the biochemical and molecular characteristics needed to die via apoptosis.

In summary, this study shows that sevoflurane improves neurological outcome after severe cerebral ischemia in rats compared with animals anesthetized with zoletil. Longer duration of cerebral ischemia is associated with more neuronal damage than shorter durations of ischemia.

\section{Acknowledgements}

Supported by grant to 04-2007-002 from the SNUBH Research Fund.

\section{References}

1. Baughman VL, Hoffman WE, Miletich DJ, Albrecht RF, Thomas C. Neurologic outcome in rats following incomplete cerebral ischemia during halothane, isoflurane, or $\mathrm{N}_{2} \mathrm{O}$. Anesthesiology 1988; 69: 192-8.

2. Engelhard K, Werner C, Reeker W, Lu H, Mollenberg O, Mielke L, et al. Desflurane and isoflurane improve neurological outcome after incomplete cerebral ischaemia in rats. Br J Anaesth 1999; 83: 41521

3. Codaccioni JL, Velly LJ, Moubarik C, Bruder NJ, Pisano PS, Guillet 
BA. Sevoflurane preconditioning against focal cerebral ischemia: inhibition of apoptosis in the face of transient improvement of neurological outcome. Anesthesiology 2009; 110: 1271-8.

4. Werner C, Mollenberg O, Kochs E, Schulte J am Esch. Sevoflurane improves neurological outcome after incomplete cerebral ischaemia in rats. Br J Anaesth 1995; 75: 756-60.

5. Haelewyn B, Yvon A, Hanouz JL, MacKenzie ET, Ducouret P, Gerard JL, et al. Desflurane affords greater protection than halothane against focal cerebral ischaemia in the rat. Br J Anaesth 2003; 91: 390-6.

6. Miura Y, Grocott HP, Bart RD, Pearlstein RD, Dexter F, Warner DS. Differential effects of anesthetic agents on outcome from near-complete but not incomplete global ischemia in the rat. Anesthesiology 1998; 89: 391-400.

7. Smith ML, Bendek G, Dahlgren N, Rosen I, Wieloch T, Siesjo BK. Models for studying long-term recovery following forebrain ischemia in the rat. 2. A 2-vessel occlusion model. Acta Neurol Scand 1984; 69: 385-401.

8. Gionet TX, Warner DS, Verhaegen M, Thomas JD, Todd MM. Effects of intra-ischemic blood pressure on outcome from 2-vessel occlusion forebrain ischemia in the rat. Brain Res 1992; 586: 188-94.

9. Pape M, Engelhard K, Eberspacher E, Hollweck R, Kellermann K, Zintner S, et al. The long-term effect of sevoflurane on neuronal cell damage and expression of apoptotic factors after cerebral ischemia and reperfusion in rats. Anesth Analg 2006; 103: 173-9.

10. Engelhard K, Werner C, Eberspacher E, Pape M, Stegemann U, Kellermann $\mathrm{K}$, et al. Influence of propofol on neuronal damage and apoptotic factors after incomplete cerebral ischemia and reperfusion in rats: a long-term observation. Anesthesiology 2004; 101: 912-7.
11. Kawaguchi M, Furuya H, Patel PM. Neuroprotective effects of anesthetic agents. J Anesth 2005; 19: 150-6.

12. Kawaguchi M, Kimbro JR, Drummond JC, Cole DJ, Kelly PJ, Patel PM. Isoflurane delays but does not prevent cerebral infarction in rats subjected to focal ischemia. Anesthesiology 2000; 92: 1335-42.

13. Kawaguchi M, Drummond JC, Cole DJ, Kelly PJ, Spurlock MP, Patel PM. Effect of isoflurane on neuronal apoptosis in rats subjected to focal cerebral ischemia. Anesth Analg 2004; 98: 798-805.

14. Wise-Faberowski L, Raizada MK, Sumners C. Oxygen and glucose deprivation-induced neuronal apoptosis is attenuated by halothane and isoflurane. Anesth Analg 2001; 93: 1281-7.

15. Inoue S, Davis DP, Drummond JC, Cole DJ, Patel PM. The combination of isoflurane and caspase 8 inhibition results in sustained neuroprotection in rats subject to focal cerebral ischemia. Anesth Analg 2006; 102: 1548-55.

16. Engelhard K, Werner C, Eberspacher E, Pape M, Blobner M, Hutzler P, et al. Sevoflurane and propofol influence the expression of apoptosis-regulating proteins after cerebral ischaemia and reperfusion in rats. Eur J Anaesthesiol 2004; 21: 530-7.

17. Colbourne F, Li H, Buchan AM, Clemens JA. Continuing postischemic neuronal death in CA1: influence of ischemia duration and cytoprotective doses of NBQX and SNX-111 in rats. Stroke 1999; 30: 662-8.

18. Orliaguet G, Vivien B, Langeron O, Bouhemad B, Coriat P, Riou B. Minimum alveolar concentration of volatile anesthetics in rats during postnatal maturation. Anesthesiology 2001; 95: 734-9.

19. Elsersy H, Sheng H, Lynch JR, Moldovan M, Pearlstein RD, Warner DS. Effects of isoflurane versus fentanyl-nitrous oxide anesthesia on long-term outcome from severe forebrain ischemia in the rat. Anesthesiology 2004; 100: 1160-6. 